\title{
Pensamento Computacional para Alunos do Ensino Fundamental de Escolas Públicas em uma Cidade de Pequeno Porte: Um Relato e Análise de Experiência
}

\author{
José Amancio M. Santos ${ }^{1}$, Roberto A. Bittencourt ${ }^{1}$, \\ Anfranserai M. Dias ${ }^{1}$, Beatriz S. Santana ${ }^{1}$ \\ ${ }^{1}$ UEFS - Universidade Estadual de Feira de Santana \\ Av. Transnordestina, s/n, Novo Horizonte \\ Feira de Santana - BA, Brasil - 44036-900 \\ zeamancio@uefs.br, roberto@uefs.br, \\ anfranserai@ecomp.uefs.br, beatrizsantana@ieee.org
}

\begin{abstract}
The introduction of the computational thinking in the elementary schools in Brazil is still incipient. This point is even more complex in public education. In this article, we present an experience involving the third sector, government and university. The experience aims to incorporate computational thinking in elementary schools. The course was carried out in a small city with low social and economic indicators. We also present an analysis of the impact of the course on students' attitudes towards computing. The results show that, despite the low socio economic condictions, the course impacts on the interest and motivation of the students towards computing. Moreover, the institutional arrangement presented might be considered as an valuable approach to insert computational thinking into students education of public shools.
\end{abstract}

Resumo. O Brasil está atrasado no que se refere à incorporação do pensamento computacional na educação básica. O problema é agravado quando consideramos o ensino público. Neste artigo apresentamos uma experiência que envolve a articulação entre agentes do terceiro setor, poder público e universidade, em direção à inserção do pensamento computacional no ensino fundamental. $O$ curso foi realizado em uma cidade de pequeno porte e com baixos indicadores de desenvolvimento social e econômico. Também apresentamos uma análise do impacto do curso sobre atitudes dos alunos em relação à computação. Os resultados evidenciam que o ambiente desfavorável não é impedimento para estímulo e motivação dos alunos. Além disso, o arranjo institucional descrito apresenta-se como estratégia viável em cidades cujas condições geo-politicas criaram um grave cenário de estagnação social e econômica.

\section{Introdução}

O software invade hoje praticamente todos os espaços do conhecimento humano e faz com que algoritmos cheguem até mesmo a moldar diferentes aspectos da convivência humana. Neste contexto de uma sociedade digital pós-industrial, é fundamental que seus cidadãos sejam proficientes em temas como pensamento computacional [Barr et al. 2011] e fluência digital [Resnick 2012] para que não só compreendam o mundo em que vivem, mas também possam ser produtores ativos de informação e conhecimento. 
VIII Congresso Brasileiro de Informática na Educação (CBIE 2019)

Anais do XXV Workshop de Informática na Escola (WIE 2019)

Enquanto países como Estados Unidos, Israel, Nova Zelândia, Reino Unido e outros formalizam gradualmente a curricularização da computação na educação básica [Schulte et al. 2012, Garneli et al. 2015], o Brasil ainda padece de uma compreensão por parte das autoridades competentes para a incorporação da computação na escola. A Sociedade Brasileira de Computação tem procurado reduzir esta visão através da definição de referenciais curriculares para computação na educação básica [SBC 2017] e da ação política junto ao Ministério da Educação e ao Conselho Nacional de Educação.

Quando analisamos a rede pública de educação básica, a dificuldade de operacionalização de iniciativas de ensino-aprendizagem de computação é ainda maior, seja pela ausência de professores da área ou pela inexistência ou precariedade da infraestrutura necessária [Bordini et al. 2016]. É neste contexto que este trabalho se insere. De que maneira as iniciativas da comunidade acadêmica de educação em computação podem ser internalizadas nas escolas públicas brasileiras? Como formalizar a formação em pensamento computacional em escolas do ensino fundamental? Não são poucas as ações da comunidade neste sentido. Num mapeamento sistemático sobre iniciativas para o ensino de pensamento computacional e programação, Santos et al. (2018) identificaram 338 artigos sobre o assunto entre 2001 e 2016, sendo que 140 deles descrevem atividades no ensino fundamental. Entretanto, a maioria destas atividades ocorre na forma de atividades pontuais, desligadas do currículo escolar. Para serem integradas ao currículo formal, é preciso projetar currículos escolares que envolvam a computação e trabalhar com materiais didáticos adequados ao nível educacional de estudantes do ensino fundamental.

Este trabalho é uma iniciativa na direção de formalização do ensino-aprendizagem de computação no ensino fundamental. A partir da abordagem de computação criativa de Karen Brennan (2013), da Universidade de Harvard [Brennan and Resnick 2012], e do uso do ambiente de aprendizagem lúdico Scratch [Resnick et al. 2009], realizamos um curso de computação criativa para estudantes do oitavo e nono anos da rede escolar pública de Piritiba, município do interior da Bahia. Este artigo relata esta experiência e analisa o seu impacto sobre as atitudes dos alunos em relação à computação.

\section{Fundamentação Teórica}

Segundo Wing (2006), o pensamento computacional (PC) é um processo envolvido na formulação e resolução de problemas que utiliza técnicas advindas da computação e é considerado uma habilidade fundamental não apenas para cientistas, mas para todos [SBC 2017]. Esta competência faz parte do processo natural do ser humano e está fortemente relacionada a outras habilidades, como ler, escrever e realizar operações matemáticas [Brennan and Resnick 2012, Wing 2011, Wing 2006, Barr et al. 2011, Barr and Stephenson 2011].

Organizações profissionais, universidades, a indústria, além dos próprios governos, demonstram um interesse acentuado e crescente na incorporação do PC nos currículos desde as séries iniciais em escolas básicas dos Estados Unidos e Reino Unido, como descreve Wing (2011) . Essas instituições entendem que os benefícios do pensamento computacional podem ser transferidos para qualquer domínio e são estratégicos para o desenvolvimento de uma nação [Brackmann et al. 2016, Seehorn et al. 2011]. A Sociedade Brasileira de Computação reforça essa ideia: "não é mais possível imaginar uma sociedade na qual os indivíduos não necessitem conhecimentos básicos de 
VIII Congresso Brasileiro de Informática na Educação (CBIE 2019)

Anais do XXV Workshop de Informática na Escola (WIE 2019)

Computação, tão importantes para a vida na sociedade contemporânea quanto os conhecimentos básicos" [SBC 2017].

Resnick (2007) expõe uma forma amigável de realizar a introdução do pensamento computacional no ensino básico por meio da computação criativa. Neste processo, o estudante utiliza conceitos natos, observados em turmas de jardim da infância, como imaginar, criar, compartilhar e refletir para o aprendizado da computação e o desenvolvimento de habilidades desejáveis, no presente século, possibilitado pelo Scratch, uma ambiente visual de aprendizagem baseado no conceito de blocos de montar [Resnick et al. 2009].

No Brasil, há algumas iniciativas para a disseminação do ensino de computação, mas elas são incipientes [de França and do Amaral 2013, Oliveira et al. 2014]. Medeiros et al. (2018) utilizam-se da computação desplugada, poemas literários e a linguagem de programação Python como método de aprendizagem. Os autores descrevem oficinas realizadas com alunos do sexto ano, em uma cidade de Rio Grande do Norte. Na oficina, apesar da curta duração da intervenção, as percepções da professora de português demonstram um impacto positivo no comportamento e rendimento dos alunos. Já Rodrigues et al. (2016) e Silva et al. (2016) relatam experiências utilizando o Scratch como facilitador do processo. Ambos utilizaram a ferramenta para a criação de jogos e observaram bons resultados relativos à experiência dos estudantes, tais como: desenvolvimento da criatividade e do pensamento sistemático, melhoria do trabalho em grupo, entre outros. Apesar dessas iniciativas, Santos et al. (2018) afirmam que são necessárias melhorias relacionadas à qualidade e profundidade dos estudos que vêm sendo apresentados.

\section{A experiência da Computação Criativa em Piritiba}

A experiência que descrevemos foi realizada na cidade de Piritiba, localizada na região centro-norte do estado da Bahia, a $320 \mathrm{Km}$ da capital Salvador. A cidade possui baixos indicadores sociais e uma população de aproximadamente 25 mil habitantes, que permanece praticamente inalterada por décadas devido às poucas condições para formação e fixação de jovens em um mercado local ou regional. Considerando o IDEB ${ }^{1}$, Piritiba está entre as últimas colocações na Bahia: dentre os 404 municípios do estado que apresentam resultados no IDEB de 2017, para os anos finais do ensino fundamental ( $8^{\mathrm{a}}$ série $/ 9^{\circ}$ ano) das escolas públicas, Piritiba ocupa a posição 393. O cenário torna-se mais alarmante quando observamos que a Bahia está abaixo das metas estabelecidas e está na última colocação dentre os estados do Brasil, juntamente com Rio Grande do Norte e Sergipe.

O curso de computação criativa realizado em Piritiba foi viabilizado a partir de um arranjo envolvendo três instituições? 2 ; o Instituto Tecnológico de Piritiba (ITPi), a Universidade Estadual de Feira de Santana (UEFS) e a Prefeitura Municipal de Piritiba (PMP). O ITPi atuou como articulador e responsável pela estruturação do projeto na cidade de Piritiba, disponibilizando de espaço físico e serviços de coordenação e secretariado. Através de um projeto de extensão, professores da UEFS forneceram know-how, traduzindo e adaptando o curso de Computação Criativa disponibilizado pela Universidade de Harvard para jovens, e também preparando o professor-facilitador do município. A PMP cedeu o professor-facilitador, que é funcionário da prefeitura.

\footnotetext{
${ }^{1}$ IDEB é o principal indicador utilizado pelo governo federal para avaliar a qualidade do ensino nas escolas do ensino básico (http://ideb.inep.gov.br/)

2 http://itpi.org.br,http://uefs.br,http://piritiba.ba.gov.br/
} 
VIII Congresso Brasileiro de Informática na Educação (CBIE 2019)

Anais do XXV Workshop de Informática na Escola (WIE 2019)

\subsection{Planejamento do curso}

O curso de Computação Criativa, traduzido e adaptado para português pelos professores da UEFS, está disponível no site da Universidade de Harvard ${ }^{3}$ O curso possui 7 unidades. Por restrições de espaço, a Tabela 1 apresenta um resumo da descrição de cada unidade.

Unidade 0. Cultura da computação criativa; infraestrutura técnica (criar contas, iniciar diários) e social (estabelecer grupos de discussão) do Scratch

Unidade 1. Conceito computacional de sequência - um tutorial passo a passo, um desafio criativo usando um número limitado de blocos, e explorações abertas por meio de um projeto sobre si mesmo

Unidade 2. Recursos visuais e de áudio em atividades focadas em animação, arte e música. Conceitos computacionais de loops, eventos e paralelismo - criando sua própria banda, criaturas animadas e um videoclipe para uma música favorita

Unidade 3. Criação de narrativas colaborativas. Desenvolver personagens: codificar conversas e inseri-los em cenas. Combinar personagens e recriar histórias

Unidade 4. Mecânicas de jogo, como pontuação e níveis, usando conceitos de variáveis, operadores e condicionais. Criação de jogos e implementação de jogos clássicos, como o Pong

Unidade 5. Revisitar trabalhos anteriores, explorando os conceitos avançados ou ajudando os outros, projetando novas atividades ou depurando desafios

Unidade 6. Projetar e desenvolver um projeto próprio por meio de ciclos iterativos de planejamento, criação e compartilhamento.

\section{Tabela 1. Descrição das unidades do curso de Computação Criativa disponibili- zado pela Universidade de Harvard e adotado em Piritiba}

O curso completo tem uma carga horária de aproximadamente 40 horas. Em Piritiba, devido a restrições de tempo e recursos, o curso teve duração de 30 horas. A principal restrição foi com relação à Unidade 5, que requer uso de extensões de hardware que não estavam disponíveis. A Unidade 6 prepara os estudantes para a realização de uma hackathon. Neste caso, apenas parte das práticas foi realizada por limitações de tempo. As aulas ocorreram entre agosto e dezembro de 2018.

\subsection{Coleta de dados para análise}

A coleta de dados foi realizada em três etapas, descritas a seguir:

1. Na matrícula, Os pais dos alunos assinaram um termo de consentimento livre e esclarecido, autorizando a realização da pesquisa.

2. Os participantes preencheram um questionário de caracterização na culminância do curso, um evento em que todos apresentaram seus trabalhos finais. $\mathrm{O}$ objetivo deste questionário foi identificar o perfil dos participantes com relação ao uso de computadores e Internet. Este questionário foi elaborado a partir dos trabalhos apresentados por Bittencourt et al. (2015) e Liebenberg et al. (2015) .

3. Logo em seguida ao preenchimento do questionário de caracterização, os participantes responderam ao questionário de avaliação de atitudes. Para isso, adotamos construtos relacionados a confiança e interesse do questionário proposto por Hoegh e Moskal (2009). Utilizamos 5 níveis de concordância e emojis para simplificar a comunicação. Além disso, realizamos uma adaptação devido ao fato de termos aplicado o questionário no momento da culminância do curso: adicionamos a frase "Por causa do curso de computação criativa, ..." antes dos construtos. Na Figura 1, apresentamos uma das questões para exemplificar o formato adotado.

${ }^{3}$ Ver Seção translation em http://creativecomputing.gse.harvard.edu/guide/ curriculum.html 
VIII Congresso Brasileiro de Informática na Educação (CBIE 2019)

Anais do XXV Workshop de Informática na Escola (WIE 2019)

1. Por causa do curso de computação criativa, eu me sinto confortável em aprender conceitos de computação.
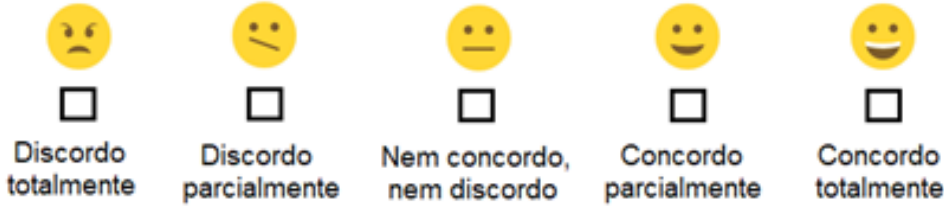

Figura 1. Exemplo de questão do formulário de avaliação

\subsection{Estratégia de avaliação}

A análise que apresentamos tem o objetivo de avaliar o impacto do curso com relação às atitudes dos participantes em relação à computação. Avaliações desta natureza têm sido adotada em vários trabalhos [Heersink and Moskal 2010, Yadav et al. 2014, Bittencourt et al. 2015]. Adotamos uma análise quantitativa baseada em questionários validados na área [Hoegh and Moskal 2009]. Com base nas respostas, agrupamos as questões de acordo com 3 tópicos principais: interesse, confiança e expectativa do uso de computação no futuro. Adotamos uma escala de Likert para analisar as respostas dos participantes.

\section{Resultados}

O curso iniciou com 45 alunos matriculados e teve 23 concluintes. A taxa de evasão foi considerada alta e esta discussão será abordada na Seção 5. Dentre os alunos que finalizaram o curso, 17 participaram da pesquisa respondendo os questionários solicitados: 4 do sexo masculino e 13 do sexo feminino. Os alunos tinham, em sua maioria, entre $14 \mathrm{e}$ 15 anos. A Tabela 2 apresenta a distribuição a distribuição de acordo com a idade.

\begin{tabular}{l|c|c|c|c}
\hline Idade (anos) & 13 & 14 & 15 & 16 \\
\hline Qtde de alunos & 1 & 5 & 10 & 1 \\
\hline
\end{tabular}

Tabela 2. Idade dos alunos

O cenário observado demonstra que o contato dos alunos com o universo digital é bastante limitado. Dos respondentes, 15 dos 17 afirmaram usar muito a Internet ("todos os dias" ou "quase todos os dias"). Quatorze deles o faziam especialmente através do celular ou tablet, sugerindo pouco domínio do uso de computadores para a maioria dos alunos. Todos usavam a Internet para fazer buscas, mas faziam pouco uso de dicionários ou enciclopédias digitais. Todos faziam muito uso de redes sociais, mas pouco uso de email ou videoconferências (e.g., Skype). Outro ponto também observado que evidencia o distanciamento dos alunos com habilidades específicas da computação é que criar programas ou dispositivos não faz parte da realidade de praticamente a totalidade dos respondentes.

\subsection{Análise do impacto do curso nas atitudes sobre computação dos alunos}

A análise apresentada nessa seção foi extraída das respostas do questionário de avaliação de atitudes. A Tabela 3 contém as questões que foram utilizadas.

O primeiro tópico desta análise quantifica o interesse dos alunos em relação à computação. O gráfico apresentado na Figura 2 resume as respostas dadas às questões 
VIII Congresso Brasileiro de Informática na Educação (CBIE 2019)

Anais do XXV Workshop de Informática na Escola (WIE 2019)

Por causa do curso de Computação Criativa...

Q4.1 - ...eu me sinto confortável em aprender conceitos de computação.

Q4.2 - ...eu posso aprender a entender conceitos de computação.

Q4.3 - ...estou confiante de que posso resolver problemas usando programas computador.

Q4.4 - ...espero que minha carreira futura exija o uso de conceitos de computação.

Q4.5 - ...eu gosto de usar a computação para resolver problemas.

Q4.6 - ...o desafio de resolver problemas usando a ciência da computação me atrai.

Q4.7 - ...eu acho que a computação é interessante.

Q4.8 - ...eu participaria voluntariamente de disciplinas de computação se me fosse dada a oportunidade.

\section{Tabela 3. Questões aplicadas}

Q4.7 e Q4.8. Os dados indicam que 94,1\% dos alunos afirmam que passaram a achar a computação interessante pela influência do curso de computação criativa (questão 4.7). E 94,2\% dos alunos afirmam estar dispostos a se envolverem com mais atividades nesta área de conhecimento (questão 4.8). Estes altos valores percentuais de concordância evidenciam impacto positivo do curso com relação ao interesse dos alunos pela computação.

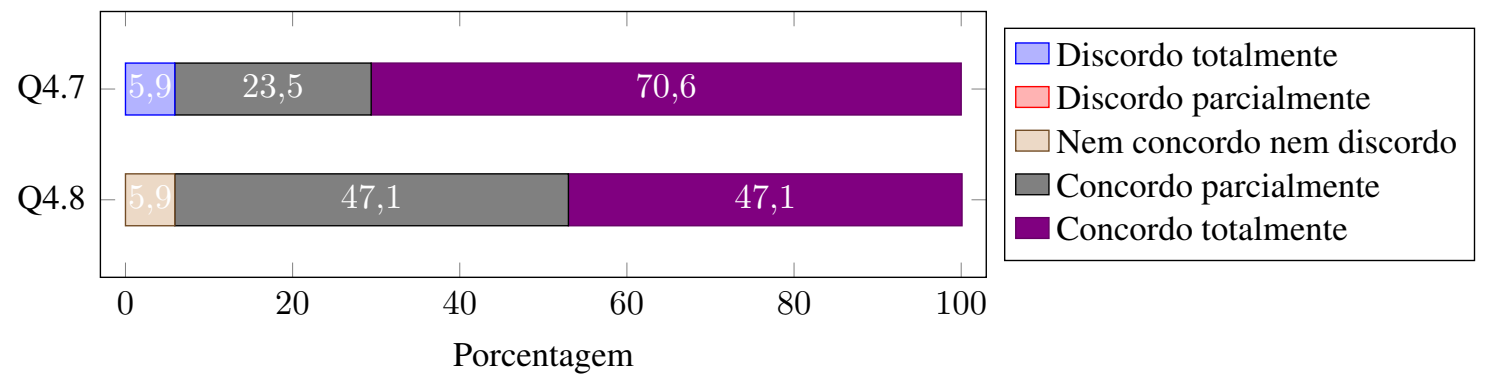

Figura 2. Respostas referente ao tópico interesse em computação

Também é alto o percentual de alunos que concordam que o curso cria uma base de conhecimento para a computação. A Figura 3 apresenta as respostas relacionadas a este tópico. Na figura, é possível observar que $82,4 \%$ dos participantes concordam total ou parcialmente que, por causa do curso, se sentem mais confortáveis em aprender conceitos de computação (questão 4.1). Além disso, 70,6\% indicam que se sentem confortáveis para entender melhor os conceitos de computação (questão 4.2).

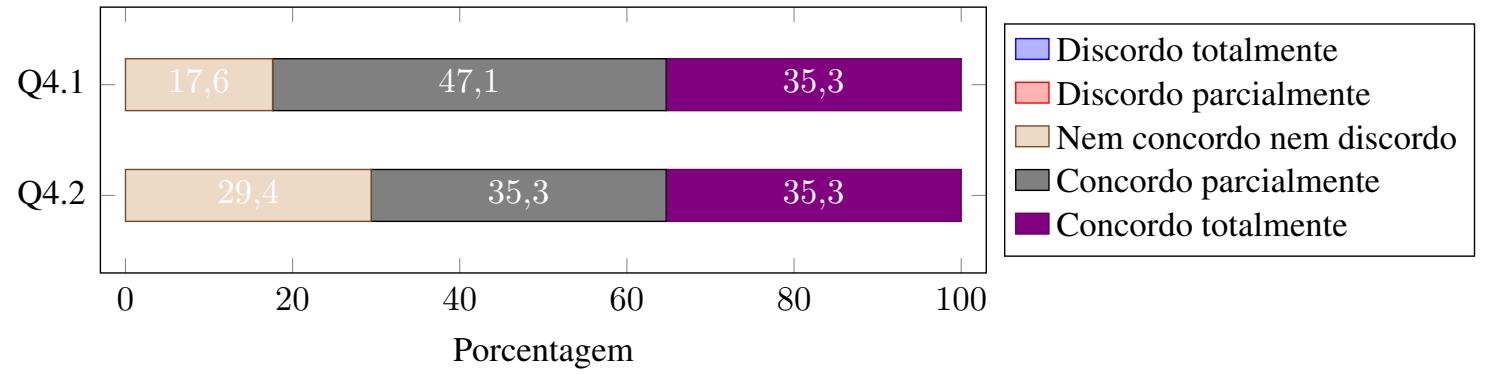

Figura 3. Base para a computação

Com relação ao estímulo para escolha de uma profissão relacionada à computação, vale destacar que este não é o objetivo do curso. Além disso, a idade dos participantes não coloca esta questão como uma das relevantes a serem observadas. Dessa forma, 
VIII Congresso Brasileiro de Informática na Educação (CBIE 2019)

Anais do XXV Workshop de Informática na Escola (WIE 2019)

quando classificamos as questões para este tema, observamos tanto a concordância quanto a discordância declarada pelos participantes. Os resultados são apresentados na Figura 4. Enquanto 58,8\% dos participantes concordam total ou parcialmente com a declaração de se sentir atraídos pelo desafio de resolver problemas da ciência da computação (questão 4.6), apenas $23,5 \%$ discordam total ou parcialmente. Da mesma forma, $58,8 \%$ dos participantes declaram concordar total ou parcialmente que gostam de usar computação para resolver problemas (questão 4.5), enquanto apenas 11,8\% discordam total ou parcialmente com esta declaração. A questão 4.3 aborda a confiança dos estudantes com relação a resolver problemas usando programas de computador. Neste caso, apenas $17,7 \%$ discordam total ou parcialmente. Finalmente, a questão 4.4 explicita a questão da escolha de uma carreira futura ligada à computação quando indica ser esta a expectativa do aluno, em função do curso de computação criativa. Neste caso, apenas 5,9\% (que corresponde a 1 aluno) indicou discordar parcialmente da questão. A maioria das respostas foi neutra (nem concordo, nem discordo), com 47,1\%. De certa forma, isso reforça o argumento de que este tópico não é foco do curso, seja pelo seu objetivo ou pela idade dos alunos.

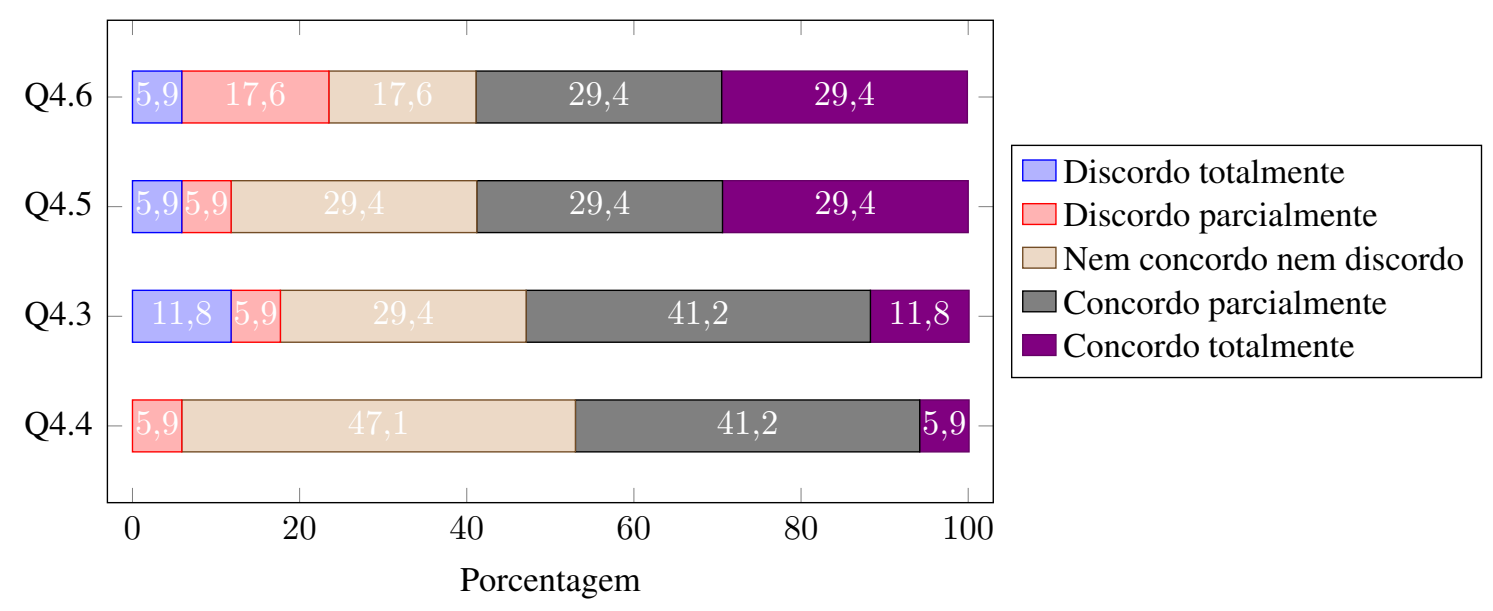

Figura 4. Estímulo para a computação

\section{Discussão}

O primeiro aspecto a ser destacado pela experiência e informações apresentadas nas Seções 3 e 4 é a viabilidade da proposição de uma formação em Pensamento Computacional mesmo em um ambiente desfavorável. Nossa experiência foi realizada no contexto de uma educação pública com baixos indicadores de qualidade, com alunos de baixa renda e que fazem uso do universo digital basicamente para acesso a redes sociais. Além disso, na maioria das vezes não utilizam computadores-alguns nem internet acessam diariamente. No entanto, o fato da maioria dos alunos demonstrarem interesse e se sentirem estimulados e confortáveis para lidar com a computação (ver Figuras 2, 3, e 4) é um indício de que condições desfavoráveis não são impedimento para o desenvolvimento de habilidades relacionadas ao Pensamento Computacional no contexto apresentado.

Um segundo aspecto está relacionado à conjuntura socio-econômica de municípios de pequeno porte e com pouco desenvolvimento econômico e social. Nestes casos, o arranjo institucional apresentado tem potencial para viabilizar uma formação mais apropriada para as demandas dos dias atuais. A atuação do denominado terceiro setor 
VIII Congresso Brasileiro de Informática na Educação (CBIE 2019)

Anais do XXV Workshop de Informática na Escola (WIE 2019)

(ITPi) promovendo articulação entre universidade (UEFS) e o poder público (Prefeitura de Piritiba) cria um ambiente onde cada parte colabora com sua habilidade para avanços em um projeto de educação que deve ser responsabilidade de toda a sociedade. Devido à boa aceitação dessa mobilização pelos agentes envolvidos e pela população local e regional, a história dessa articulação foi descrita em um portal de notícias importante do estado da Bahia [Santos and Barbosa 2018]. Uma evidência do seu sucesso está na ampliação das turmas de formação em Computação Criativas em Piritiba. A partir do sucesso da experiência apresentada neste artigo, o curso está sendo aplicado em 2019 para 83 alunos, divididos em 6 turmas. A secretaria de educação do município incorporou o curso como parte de atividades complementares das escolas, da mesma forma que disponibiliza reforço de português e matemática, por exemplo.

Um terceiro aspecto a ser observado é o efeito positivo da experiência adquirida pelas pessoas envolvidas no projeto. Neste caso destacamos as observações realizadas durante as práticas. Por exemplo, no curso realizado em 2018 as turmas continham alunos do sétimo e oitavo ano do ensino fundamental pela compreensão da equipe de que os alunos mais novos não teriam condições de acompanhar as atividades propostas. Entretanto, percebemos que o ambiente desfavorável não impactou na capacidade dos alunos em acompanhar as práticas e em 2019 o curso está sendo realizado para alunos do sexto e sétimo ano. Outra questão relevante está relacionada à evasão. Em 2018 houve uma alta taxa de evasão (o curso iniciou com 45 alunos e terminou com 23). Em 2019, tem sido realizado um trabalho de sensibilização junto às famílias, o que tem impactado positivamente na frequência dos alunos. Notamos que a descrença dos familiares com relação aos benefícios que a escola pode trazer aos alunos é um fator relevante para a evasão. Este é um tema complexo, para o qual planejamos realizar uma avaliação mais sistemática.

\section{Conclusões}

Este artigo apresentou uma experiência de aprendizagem de pensamento computacional através de um curso de computação criativa com estudantes do oitavo e nono anos do ensino fundamental de uma escola pública municipal de Piritiba, município de pequeno porte no interior da Bahia. O curso teve duração de 30 horas, distribuídas entre agosto e dezembro de 2018. Vinte e três alunos concluíram o curso.

Os resultados apontam para o estímulo dos estudantes em aprender computação, a formação de uma base em computação e o uso de pensamento computacional nas vidas futuras dos participantes. A organização estabelecida sugere uma forma de interação profícua entre governo, escolas, universidade e terceiro setor, permitindo potencializar a introdução da computação na educação básica. O arranjo realizado pode ser reproduzido em outros locais ou adaptado às condições específicas do ambiente político e acadêmico.

Pretendemos aplicar os materiais didáticos de computação criativa com estudantes mais novos, especialmente do sexto e sétimo anos do ensino fundamental. Além disso, nosso grupo de pesquisa está criando um currículo para o ensino fundamental II e para o ensino médio. Finalmente, pretendemos realizar avaliações mais pormenorizadas das experiências educacionais do ponto de vista de aprendizagem, atitudes, motivação e engajamento dos estudantes participantes deste tipo de intervenção. 
VIII Congresso Brasileiro de Informática na Educação (CBIE 2019)

Anais do XXV Workshop de Informática na Escola (WIE 2019)

\section{Referências}

[Barr et al. 2011] Barr, D., Harrison, J., and Conery, L. (2011). Computational Thinking: A Digital Age Skill for Everyone. Learning \& Leading with Technology, 38(6):20-23.

[Barr and Stephenson 2011] Barr, V. and Stephenson, C. (2011). Bringing Computational Thinking to K-12: What is Involved and What is the Role of the Computer Science Education Community? ACM Inroads, 2(1):48-54.

[Bittencourt et al. 2015] Bittencourt, R. A., Santos, D. M. B., Rodrigues, C. A., Batista, W. P., and Chalegre, H. S. (2015). Learning Programming with Peer Support, Games, Challenges and Scratch. In FIE 2015 - IEEE Frontiers in Education Conference.

[Bordini et al. 2016] Bordini, A., Avila, C. M. O., Weisshahn, Y., da Cunha, M. M., da Costa Cavalheiro, S. A., Foss, L., Aguiar, M. S., and Reiser, R. H. S. (2016). Computaçao na Educaçao Básica no Brasil: O Estado da Arte. Revista de Informática Teórica e Aplicada, 23(2):210-238.

[Brackmann et al. 2016] Brackmann, C., Barone, D., Casali, A., Boucinha, R., and MuñozHernandez, S. (2016). Computational thinking: Panorama of the Americas. In SIIE, pages $1-6$. IEEE.

[Brennan and Resnick 2012] Brennan, K. and Resnick, M. (2012). New Frameworks for Studying and Assessing the Development of Computational Thinking. In Proceedings of the 2012 annual meeting of the AERA, Vancouver, Canada, volume 1, page 25.

[da Silva et al. 2016] da Silva, G. T., de Souza, J. L., and da Silva, L. A. M. (2016). Aplicação da Ferramenta Scratch para o Aprendizado de Programação no Ensino Fundamental I. In Anais dos Workshops do CBIE.

[de França and do Amaral 2013] de França, R. S. and do Amaral, H. J. C. (2013). Ensino de Computaçao na Educaçao Básica no Brasil: Um Mapeamento Sistemático. In XXI Workshop sobre Educaçao em Computaçao.

[Garneli et al. 2015] Garneli, V., Giannakos, M. N., and Chorianopoulos, K. (2015). Computing Education in K-12 Schools: A Review of the Literature. In EDUCON, pages 543-551. IEEE.

[Heersink and Moskal 2010] Heersink, D. and Moskal, B. M. (2010). Measuring High School Students' Attitudes toward Computing. In Proceedings of the 41st ACM SIGCSE Technical Symposium, pages 446-450. ACM.

[Hoegh and Moskal 2009] Hoegh, A. and Moskal, B. M. (2009). Examining Science and Engineering Students' Attitudes toward Computer Science. In Proceedings of the 39th IEEE international conference on FIE, pages 1306-1311. IEEE Press.

[Liebenberg et al. 2015] Liebenberg, J., Huisman, M., and Mentz, E. (2015). The Relevance of Software Development Education for Students. IEEE ToE, 58(4):242-248.

[Medeiros et al. 2018] Medeiros, S., Rabelo, H., Garcia, T. C. M., Nunes, I., Medeiros, F., Medeiros, A., and de Souza Rabelo, D. S. (2018). Ensino de Algoritmos Através de Poesia Compilada e Computação Desplugada: Relato de Experiência com Alunos de Ensino Fundamental. In Anais do WIE, page 381. 
VIII Congresso Brasileiro de Informática na Educação (CBIE 2019)

Anais do XXV Workshop de Informática na Escola (WIE 2019)

[Oliveira et al. 2014] Oliveira, M. d., Souza, A. d., Ferreira, A., and Barbosa, E. (2014). Ensino de Lógica de Programação no Ensino Fundamental Utilizando o Scratch: Um Relato de Experiência. In XXXIV CSBC-Workshop de Ensino de Computação, Brasília.

[Resnick 2007] Resnick, M. (2007). All I Really Need to Know (About Creative Thinking) I Learned (by Studying How Children Learn) in Kindergarten. In Proceedings of the 6th Conference on Creativity \& Cognition, C\&C '07, pages 1-6, New York, NY, USA. ACM.

[Resnick 2012] Resnick, M. (2012). Point of View: Reviving Papert's Dream. Educational Technology, 52(4):42.

[Resnick et al. 2009] Resnick, M., Maloney, J., Monroy-Hernández, A., Rusk, N., Eastmond, E., Brennan, K., Millner, A., Rosenbaum, E., Silver, J., Silverman, B., et al. (2009). Scratch: Programming for All. Communications of the ACM, 52(11):60-67.

[Rodrigues et al. 2016] Rodrigues, L. C., Queiroga, A., Oliveira, M., and More, A. (2016). Relato de Experiência: Curso de Introdução à Programação para Crianças do Ensino Fundamental no IFSP Votuporanga. In Anais do WIE, page 349.

[Santos and Barbosa 2018] Santos, J. A. M. and Barbosa, T. A. (2018). Instituto Tecnológico de Piritiba Leva Curso de Computação Criativa Idealizado pela Universidade de Harvard para o Sertão da Bahia, Portal Bahia Notícias: https://goo.gl/k1W46D.

[Santos et al. 2018] Santos, P. S. C., Araujo, L. G. J., and Bittencourt, R. A. (2018). A Mapping Study of Computational Thinking and Programming in Brazilian K-12 Education. In FIE 2018 - IEEE Frontiers in Education Conference, pages 1-8. IEEE.

[SBC 2017] SBC (2017). Referenciais de Formação em Computação: Educação Básica. http://www.sbc.org.br/files/ ComputacaoEducacaoBasica-versaofinal-julho2017.pdf.

[Schulte et al. 2012] Schulte, C., Hornung, M., Sentance, S., Dagiene, V., Jevsikova, T., Thota, N., Eckerdal, A., and Peters, A.-K. (2012). Computer Science at School/CS Teacher Education: Koli Working-Group Report on CS at School. In Proceedings of the 12th Koli Calling International Conference on Computing Education Research, pages 29-38. ACM.

[Seehorn et al. 2011] Seehorn, D., Carey, S., Fuschetto, B., Lee, I., Moix, D., O'GradyCunniff, D., Owens, B. B., Stephenson, C., and Verno, A. (2011). CSTA K-12 Computer Science Standards: Revised 2011. Technical report, CSTA/ACM, New York, NY, USA. 104111.

[Wing 2011] Wing, J. (2011). Research Notebook: Computational Thinking - What and Why. The Link Magazine, pages 20-23.

[Wing 2006] Wing, J. M. (2006). Computational Thinking. Communications of the ACM, 49(3):33-35.

[Yadav et al. 2014] Yadav, A., Mayfield, C., Zhou, N., Hambrusch, S., and Korb, J. T. (2014). Computational Thinking in Elementary and Secondary Teacher Education. Trans. Comput. Educ., 14(1):5:1-5:16. 